\title{
A comparison of analgesic efficacy between oblique subcostal transversus abdominis plane block and intravenous morphine for laparascopic cholecystectomy. A prospective randomized controlled trial
}

\author{
Chee Kean Chen ${ }^{1,2}$, Peter Chee Seong Tan ${ }^{2}$, Vui Eng Phui ${ }^{2}$, and Shu Ching Teo ${ }^{2}$ \\ ${ }^{1}$ Department of Anesthesiology and Intensive Care, Normah Medical Specialist Centre, ${ }^{2}$ Department of Anesthesiology, Sarawak \\ General Hospital, Kuching, Sarawak, Malaysia
}

Background: The ultrasound-guided oblique subcostal transversus abdominis plane (OSTAP) block provides a wider area of sensory block to the anterior abdominal wall than the classical posterior approach. We compared the intra-operative analgesic efficacy of OSTAP block with conventional intravenous (IV) morphine during laparoscopic cholecystectomy.

Methods: Forty adult patients undergoing laparoscopic cholecystectomy under standard general anesthesia, were randomly assigned for either bilateral OSTAP block using $1.5 \mathrm{mg} / \mathrm{kg}$ ropivacaine on each side $(\mathrm{n}=20)$ or IV morphine $0.1 \mathrm{mg} / \mathrm{kg}(\mathrm{n}=20)$. The intra-operative pulse rate, systolic and diastolic blood pressure and mean arterial blood pressure were monitored every five minutes. Repetitive boluses of IV fentanyl $0.5 \mu \mathrm{g} / \mathrm{kg}$ were given as rescue analgesia when any of the above-mentioned parameters rose more than $15 \%$ from the baseline values. Time to extubation was documented. Additional boluses of IV morphine $0.05 \mathrm{mg} / \mathrm{kg}$ were administered in the recovery room if the recorded visual analogue score (VAS) was more than 4 . Nausea and vomiting score, as well as sedation score were recorded.

Results: The morphine group required more rescue fentanyl as compared to the OSTAP block group but the difference was not significant statistically. Time to extubation was significantly shorter in the OSTAP block group (mean [SD] 10.4 [2.60] vs 12.4 [2.54] min; $\mathrm{P}=0.021$ ). Both methods provided excellent analgesia and did not differ in postoperative morphine requirements. No between-group differences in sedation score and incidence of nausea and vomiting were demonstrated.

Conclusions: Ultrasound-guided OSTAP block has an important role as part of balanced anesthesia. It is as efficacious as IV morphine in providing effective analgesia during laparoscopic cholecystectomy. (Korean J Anesthesiol 2013; 64: 511-516)

Key Words: Laparascopic cholecystectomy, Oblique subcostal transversus abdominis plane block, Ultrasoundguided regional anesthesia.

Received: September 14, 2012. Revised: 1st, October 22, 2012; 2nd, November 2, 2012. Accepted: November 13, 2012.

Corresponding author: Chee Kean Chen, M.D., Department of Anesthesiology and Intensive Care, Normah Medical Specialist Centre, Jalan Tun Abdul Rahman, Petra Jaya, Kuching, Sarawak 93050, Malaysia. Tel: 60-82440055, Fax: 60-82442600, E-mail: chenck@hotmail.my

This paper had been performed a poster presentation at the International Symposium on Spine and Paravertebral Sonography, March 29-April 1, 2012, Shatin, Hong Kong.

두 This is an open-access article distributed under the terms of the Creative Commons Attribution Non-Commercial License (http:// creativecommons.org/licenses/by-nc/3.0/), which permits unrestricted non-commercial use, distribution, and reproduction in any medium, provided the original work is properly cited. 


\section{Introduction}

Anterior abdominal pain is common and it causes significant morbidity among patients undergoing abdominal surgery [1]. The transversus abdominis plane (TAP) block is a regional anesthesia technique where local anesthetic is injected into the neurovascular plane between the transversus abdominis and internal oblique muscles in the anterolateral abdominal wall. The classical or posterior TAP block which involves the injection of local anesthetic at the anterior axillary line between the lower costal margin and iliac crest, provides sensory block below the umbilicus [2,3]. Various studies have demonstrated the efficacy of TAP block in providing effective postoperative analgesia in patients undergoing gynecologic surgery $[4,5]$, cesarean delivery [6], appendicectomy [7] and cholecystectomy [8,9].

The oblique subcostal transversus abdominis plane (OSTAP) block is a variant of TAP block. The needle is introduced into the TAP near the costal margin, medial to the linea semilunaris, with subsequent needle advancement and hydrodissection occurring along a line from the xyphoid towards the anterior part of the iliac crest [10]. In a cadaveric study performed by Barrington and colleagues, multiple injections of TAP block similar to the oblique subcostal TAP block has been shown to provide coverage of T7-T12 dermatomes. In clinical settings, injection of a local anesthetic along the entire oblique subcostal line can result in anesthesia of T6-L1 region [11]. Recent data on this approach has also proven efficacious in providing postoperative analgesia in upper abdominal surgery [12]. Therefore the OSTAP block is suitable for surgery both superior and inferior to the umbilicus.
However, despite the encouraging initial results, there has yet to be any work comparing the intra-operative analgesic efficacy of OSTAP block with the standard systemic opioids or epidural analgesia. We hypothesize that the OSTAP blockade would have a similar efficacy as standard intravenous morphine intra- and postoperatively in patients undergoing laparascopic cholecystectomy.

\section{Materials and Methods}

After approval was granted by the Clinical Research Centre and Ethics Committee, 70 patients who were planned for elective laparascopic cholecystectomy regardless of primary pathology were screened in the surgical outpatient clinic. After fulfilling the inclusion criteria, 58 patients aged between $21-$ 60 years scheduled were enrolled. Written informed consents were obtained from these patients. Exclusion criteria were patients allergic to amino-amide local anesthetics, presence of coagulopathy, local skin infection at the needle puncture sites, and conversion of laparascopic to open surgery. The participant flow diagram is shown in Fig. 1. All patients were familiarized with visual analogue score (VAS). The VAS is a straight line of $10 \mathrm{~cm}$ in length, with one end defined as "no pain" and the other end being "excruciating pain". Patients are asked to place a mark on the line to signify the amount of pain that they are experiencing at a particular time. The distance between the end labeled "no pain" and the mark placed by the patient is measured in centimeters, to give a pain score between 0 to $10 \mathrm{~cm}$.

Patients were randomly allocated for OSTAP block or intravenous (IV) Morphine group according to a computer-generated

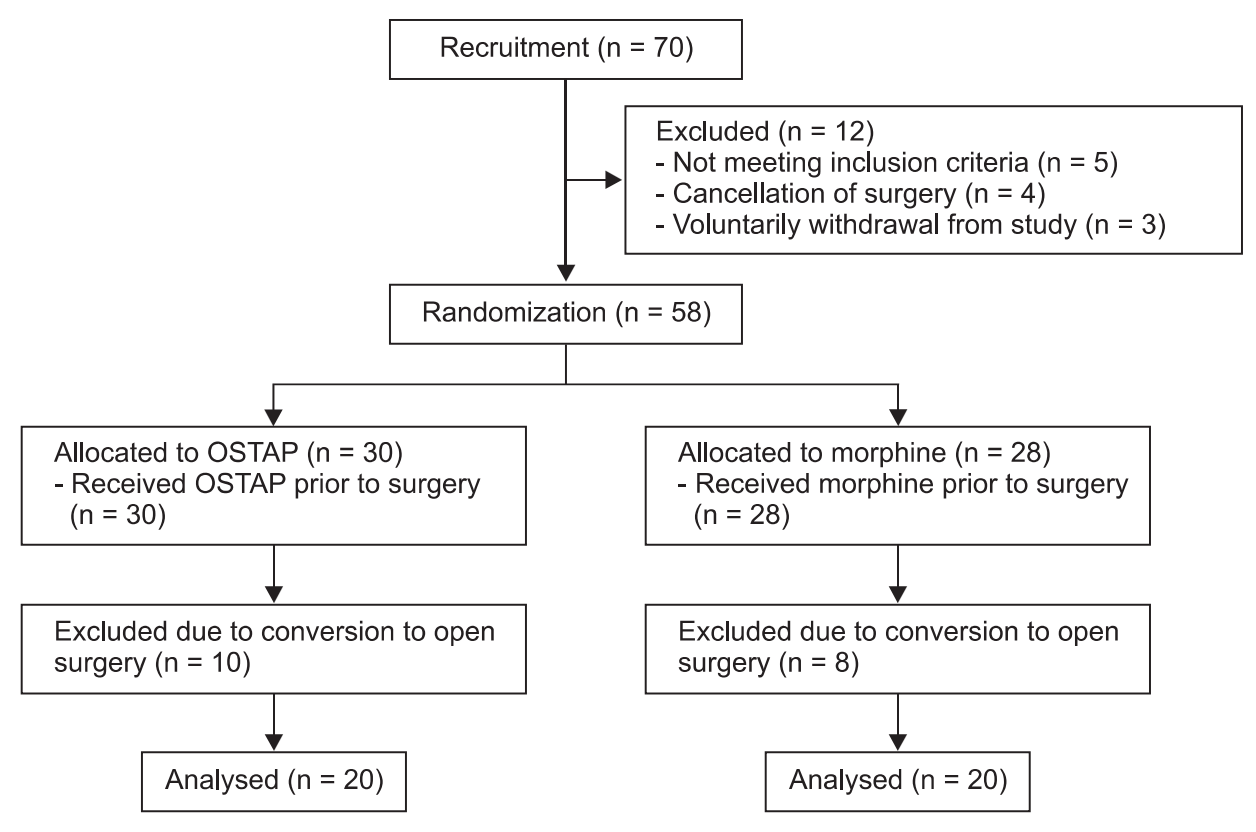

Fig. 1. Participant flow chart. 
randomization list, to obtain equal sample sizes. The patients were fasted as per protocol i.e. 6 hours for solid food and 2 hours for clear fluids before their scheduled surgeries. Premedication with oral midazolam $(7.5 \mathrm{mg}$ ) was given half an hour before induction of anesthesia. Standard monitoring was performed prior to the induction of anesthesia. Pre-induction heart rate (HR), systolic blood pressure (SBP), diastolic blood pressure (DBP) and mean arterial pressures (MAP) were recorded as baseline values. All subjects received a standard general anesthetic regimen. They were given IV fentanyl $(2 \mu \mathrm{g} /$ $\mathrm{kg}$ ), IV propofol ( $1-2 \mathrm{mg} / \mathrm{kg}$ titrations) and IV vecuronium $(0.1 \mathrm{mg} / \mathrm{kg})$ during induction. Anesthesia was maintained with sevoflurane at 1.0-1.2 minimum alveolar concentration in air/ oxygen (fractional inspired oxygen of 0.35). An intermittent dose of vecuronium $0.05 \mathrm{mg} / \mathrm{kg}$ was given to maintain adequate muscle relaxation. Mechanical ventilation was instituted using a pressure controlled mode to maintain end tidal carbon dioxide between 35 to $40 \mathrm{mmHg}$.

In the OSTAP block group, bilateral OSTAP blocks were performed aseptically under ultrasonographic guidance, with GE Logiq e ultrasound machine (GE Healthcare, United Kingdom) and a linear 5-13 MHz ultrasound transducer. The rectus abdominis and transverses abdominis muscles were identified near the costal margin and xyphoid. A 22G, $120 \mathrm{~mm}$ Stimuplex D Plus needle (B. Braun, Melsungen AG, Germany) would then be inserted through the rectus muscle 2 to $3 \mathrm{~cm}$ medial to the probe. Then, the probe was placed to image the needle in-plane. Once the tip of the needle was visualized in between the rectus muscle and transversus abdominus muscle (Fig. 2A), and negative pressure aspiration was demonstrated, $20 \mathrm{ml}$ of ropivacaine $3.75 \mathrm{mg} / \mathrm{ml}$ was administered incrementally in TAP. Hydrodissection was demonstrated by the needle passing along the oblique subcostal line inferolaterally from xyphoid towards the anterior part of the iliac crest (Fig. 2B). A contralateral OSTAP block would subsequently be performed in like manner. The total ropivacaine used was $150 \mathrm{mg}$ (or the maximum dose of $3 \mathrm{mg} / \mathrm{kg}$ ) diluted to $40 \mathrm{ml}$ with $0.9 \%$ saline solution. In order to prevent technical variants between block performers, all OSTAP block were only performed by the first investigator (C.C.K.).

The patients in the morphine group were given IV morphine of $0.1 \mathrm{mg} / \mathrm{kg}$ after induction of anesthesia. A skin incision was made 15 minutes after bilateral OSTAP blocks or IV morphine administration. The subsequent surgical procedure was performed according to the institutional standards. Independent anesthesiologists, who were not aware of the mode of intraoperative analgesia, would then complete the recording of all the hemodynamic parameters. If any of the above parameters rose by $\geq 15 \%$ relative to baseline values, IV fentanyl $0.5 \mu \mathrm{g} /$ $\mathrm{kg}$ was administered. Repeated doses of fentanyl were given every 5 minutes should these parameters remain 15\% above their baseline values. The amount of fentanyl required was documented.

After skin closure, sevoflurane was turned off and patients were given $100 \%$ oxygen with a flow rate of $8 \mathrm{~L} / \mathrm{min}$. The neuromuscular blockade was reversed with IV atropine $1 \mathrm{mg}$ and IV neostigmine $2.5 \mathrm{mg}$. Patients were extubated once they demonstrated spontaneous eye opening, good cough reflex and hand grip and were able to generate adequate tidal volume. The time required to extubate patients commenced from the time sevoflurane was turned off until the patient was extubated. The patients were observed for one hour in the recovery room. VAS score, sedation, nausea and vomiting score were recorded 30 minutes after arrival in the recovery room. Sedation was
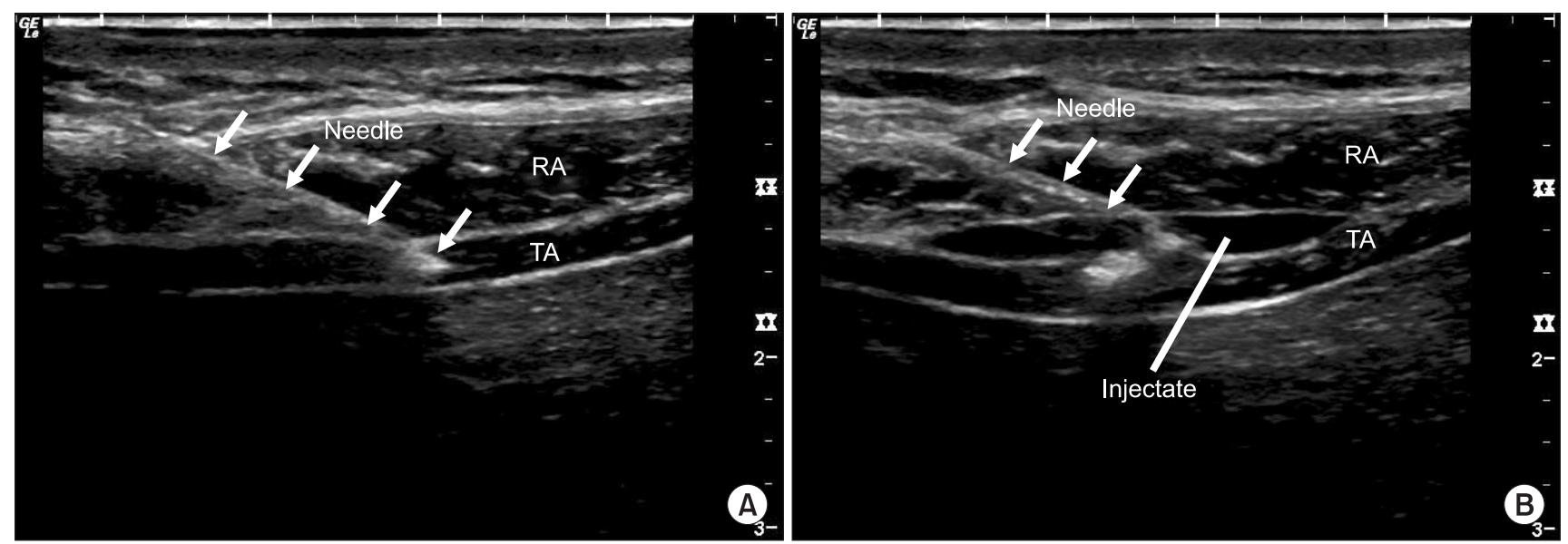

Fig. 2. (A) Ultrasonography of anterior abdomen during needle insertion. Arrows indicate the shadow of needle. (B) Ultrasonography of anterior abdomen when injectate starts to hydrodissect rectus abdominis and transversus abdominis muscles. RA: rectus abdominis, TA: transversus abdominis. 
assessed with a categorical scoring system $(0=$ awake and alert, 1 = quietly awake, 2 = asleep but easily roused, 3 = deep sleep). Nausea and vomiting were measured with a similar categorical scoring system ( $0=$ none, $1=$ mild nausea, $2=$ moderate nausea, 3 = severe nausea with vomiting). Additional boluses of IV morphine $0.05 \mathrm{mg} / \mathrm{kg}$ were given if the VAS score was more than 4 . IV metochlorpramide $10 \mathrm{mg}$ was administered to patients who had moderate or severe nausea and/or vomiting.

The primary outcome measured in this study was intraoperative fentanyl consumption. The secondary outcome measures included time required to extubate patient, postoperative morphine requirement, VAS and side effects related to morphine usage. The data was analyzed with SPSS (version 19.0, Chicago, IL, USA) in which a p-value of less than 0.05 was considered significant. The between-group differences were determined with independent t-test or Mann-Whitney test where appropriate.

\section{Results}

A total of 58 patients were randomized for intra-operative analgesic intervention. Ten patients from the OSTAP block group and 8 patients from the IV morphine group were excluded due to conversion of laparoscopic to open surgery, leaving 20 patients in each group for analysis. The demographics and perioperative details of the remaining 40 patients are presented in Table 1. There were no significant differences between the two groups in terms of patients' demographic and peri-operative data.

The IV morphine group required more rescue fentanyl as compared to the OSTAP block group but the difference was not significant statistically (Table 2). Time to extubation was significantly shorter in the OSTAP block group. Both methods delivered an excellent analgesia and did not differ in postoperative morphine consumptions. The morphine group demonstrated a higher sedation score as well as incidence of nausea and vomiting (35.3\% vs $11.8 \%$; $\mathrm{P}=0.225$ ). However, these between-group

Table 1. Demographic and Perioperative Data

\begin{tabular}{lccc}
\hline \multicolumn{1}{c}{ Variables } & $\begin{array}{c}\text { OSTAP block } \\
(\mathrm{n}=20)\end{array}$ & $\begin{array}{c}\text { IV Morphine } \\
(\mathrm{n}=20)\end{array}$ & $\mathrm{P}$ \\
\hline Age (yr) & $46.6(12.5)$ & $44.3(13.7)$ & 0.574 \\
Gender (F/M) & $15 / 5$ & $13 / 7$ & 0.490 \\
ASA (I/II) & $10 / 10$ & $10 / 10$ & 1.000 \\
Weight (kg) & $63.7(12.1)$ & $65.7(13.6)$ & 0.618 \\
Height (cm) & $157.9(9.6)$ & $159.3(11.0)$ & 0.659 \\
Body mass index $\left(\mathrm{kg} / \mathrm{m}^{2}\right)$ & $25.4(3.5)$ & $26.8(4.6)$ & 0.300 \\
Duration of surgery $\left(\mathrm{min}^{2}\right)$ & $105.4(32.9)$ & $98.7(24.9)$ & 0.472 \\
\hline
\end{tabular}

Values are given as mean (standard deviation). OSTAP block: oblique subcostal transversus abdominis plane block, IV Morphine: intravenous morphine. differences failed to show any statistical significance. There were no complications attributable to OSTAP block.

\section{Discussion}

In recent years, regional anesthesia has seen many new areas of advancement, especially with the introduction of ultrasound. With ultrasound guided anesthesia, the anatomy is clearer, and techniques are more defined and targeted. This has lead to a safer, more precise and targeted practice, with significantly reduced peri-operative complications and morbidities [13].

The rationale for selecting laparascopic cholecystectomy for this study is based on the fact that cholecystectomy is one of the most common surgeries performed. Moreover, current surgical practice is shifting towards minimally invasive techniques or keyhole surgeries, and laparascopic or even robotic surgeries are increasingly preferred by most surgeons, especially among the younger generation of surgeons [14]. Laparascopic cholecystectomy is also one of the surgeries which has a minimal fluid shift, if uncomplicated. Thus, this surgery has a minimal effect on hemodynamic swing, which in turn has a minimal influence on the fentanyl consumption. Apart from that, many previous literatures have reported that cholecystectomy is associated with a high prevalence of chronic pain $[15,16]$. With the administration of OSTAP block as preemptive anesthesia prior to surgery, the incidence of acute pain subsequently resulting in chronic pain will be significantly lower. Better outcomes in terms of reducing post-operative pain and morbidities with TAP block have been described in previous studies [4-9].

Surgical ports for laparascopic cholecystectomy are typically inserted in the para-umbilical, infra-xyphoid and epigastric areas. At times, an additional port may be placed in the area around four finger breadths below the right mid-costal line.

Table 2. Clinical Comparison of Oblique Subcostal Transversus Abdominis Plane Block and Intravenous Morphine in Laparascopic Cholecystectomy

\begin{tabular}{lccc}
\hline \multicolumn{1}{c}{ Variables } & $\begin{array}{c}\text { OSTAP block } \\
(\mathrm{n}=20)\end{array}$ & $\begin{array}{c}\text { IV Morphine } \\
(\mathrm{n}=20)\end{array}$ & $\mathrm{P}$ \\
\hline Fentanyl doses $(\mu \mathrm{g})$ & $24.5(29.1)$ & $31.3(39.2)$ & 0.540 \\
Morphine doses $(\mathrm{mg})$ & $0.4(1.0)$ & $0.0(0.0)$ & 0.080 \\
Time to extubation (min) & $10.4(2.6)$ & $12.4(2.5)$ & $0.021^{*}$ \\
Visual analogue score & $2.0(1.0)$ & $2.0(2.0)$ & 0.293 \\
Nausea vomiting score & $0.0(0.0)$ & $0.0(1.0)$ & 0.477 \\
Sedation score & $0.0(1.0)$ & $1.0(1.0)$ & 0.170 \\
\hline
\end{tabular}

Fentanyl doses, morphine doses and time to extubationare expressed as mean (standard deviation); Visual analoque score, nausea vomiting score and sedation score are expressed as median (interquartile range). OSTAP block: oblique subcostal transversus abdominis plane block, IV Morphine: intravenous morphine. *Significant P value. 
Hence, the regional anesthesia coverage required includes thoracocostal nerves 6 to 11. A study by El-Dawlatly et al. [8] demonstrated that the classical posterior TAP block significantly reduced the intra-operative sulfentanil and postoperative morphine consumption compared to the control group. With a previous cadaveric study revealing that the oblique subcostal approach has a more cephalad spread compared to the posterior approach, the approach used in our study is likely to provide adequate analgesic coverage for supra-umbilical surgeries [11].

Prior to the present study, few reports have demonstrated intra-operative efficacy of TAP block [17-19]. The outcome of this study demonstrated that OSTAP block was a reliable and effective reproducible regional anesthesia for laparascopic cholecystectomy, both intra- and post-operatively. The insignificant difference in intra-operative rescue fentanyl boluses between the two groups in this study implies that OSTAP is as efficacious as systemic opioids in laparascopic cholecystectomy. We hypothesized that OSTAP block would have a significantly lower incidence of post-operative complications, as compared to the administration of systemic opioids. However, this study has proven otherwise. An explanation for this can be due to the side effects related to morphine usage being measured as a secondary outcome for the present study. Thus, the study may not have been powered sufficiently to detect a difference in the sedation, nausea and vomiting scores for the two groups. Moreover, laparascopic cholecystectomy is a relatively short procedure and the amount of intravenous morphine given for the control group was a modest dose. The most encouraging result of OSTAP block is the significantly shorter extubation time. This implies a faster patient turnover rate which is especially beneficial in ambulatory care settings.

The drawback for this regional anesthesia is the learning curve involved and certainly the time needed to perform this block prior to surgery. This is especially so in a major service hospital, where surgeons prefer shorter induction times prior to surgery. For this approach in particular, identifying the transversus abdominis plane would be more difficult when the ultrasound probe is placed lateral to the xyphisternum, as sonography only shows two muscular layers, i.e. rectus abdominis and transversus abdominis. For beginners who perform this block, a surveillance sonography from a lateral aspect, where all three abdominal muscles are clearly shown should first be sought. Only then should the probe be moved medially toward the xyphisternum, where only two muscular layers are visible. The usage of this technique as intra-operative anaesthesia for other types of surgery remains questionable, as OSTAP block only provides anaesthesia for the anterior abdominal wall but not visceral structures [10]. Much work is needed to explore the intra-operative efficacy of this block for other types of surgery. Further study should also focus on the long term outcome of TAP block, especially the incidence of conversion of acute to chronic pain.

In conclusion, ultrasound-guided OSTAP block is safe and as efficacious as IV morphine in providing effective analgesia during laparoscopic cholecystectomy. As it is a relatively simple procedure, it has the potential role as part of a balanced anesthesia during laparascopic cholecystectomy.

\section{Acknowledgments}

The authors would like to thank Dr. Lucas Luk Tien Wee for critical review and editing support of this manuscript. The authors declare neither financial support nor any conflict of interest.

\section{References}

1. Macrae WA. Chronic pain after surgery. Br J Anaesth 2001; 87: 8898.

2. Shibata Y, Sato Y, Fujiwara Y, Komatsu T. Transversus abdominis plane block. Anesth Analg 2007; 105: 883.

3. Tran TM, Ivanusic JJ, Hebbard P, Barrington MJ. Determination of spread of injectate after ultrasound-guided transversus abdominis plane block: a cadaveric study. Br J Anaesth 2009; 102: 123-7.

4. McDonnell JG, O’Donnell B, Curley G, Heffernan A, Power C, Laffey JG. The analgesic efficacy of transversus abdominis plane block after abdominal surgery: a prospective randomized controlled trial. Anesth Analg 2007; 104: 193-7.

5. Shin HJ, Kim ST, Yim KH, Lee HS, Sim JH, Shin YD. Preemptive analgesic efficacy of ultrasound-guided transversus abdominis plane block in patients undergoing gynecologic surgery via a transverse lower abdominal skin incision. Korean J Anesthesiol 2011; 61: 413-8.

6. McDonnell JG, Curley G, Carney J, Benton A, Costello J, Maharaj $\mathrm{CH}$, et al. The analgesic efficacy of transversus abdominis block after caesarean delivery: a randomized controlled trial. Anesth Analg 2008; 106: 186-91.

7. Niraj G, Searle A, Mathews M, Misra V, Baban M, Kiani S, et al. Analgesic efficacy of ultrasound-guided transversus abdominis plane block in patients undergoing open appendicectomy. Br J Anaesth 2009; 103: 601-5.

8. El-Dawlatly AA, Turkistani A, Kettner SC, Machata AM, Delvi MB, Thallaj A, et al. Ultrasound-guided transversus abdominis plane block: Description of a new technique and comparison with conventional systemic analgesia during laparoscopic cholecystectomy. Br J Anaesth 2009; 102: 763-7.

9. Ra YS, Kim CH, Lee GY, Han JI. The analgesic effect of the ultrasound-guided transverse abdominis plane block after laparascopic cholecystectomy. Korean J Anesthesiol 2010; 58: 362-8.

10. Hebbard PD, Barrington MJ, Vasey C. Ultrasound-guided continuous oblique subcostal transversus abdominis plane blockade: description of anatomy and clinical technique. Reg Anesth Pain Med 2010; 35: 436-41. 
11. Barrington MJ, Ivanusic JJ, Rozen WM, Hebbard P. Spread of injectate after ultrasound-guided subcostal transversus abdominis plane block: a cadaveric study. Anaesthesia 2009; 64: 745-50.

12. Niraj G, Kelkar A, Jeyapalan I, Graff-Baker P, Williams O, Darbar A, et al. Comparison of analgesic efficacy of subcostal transversus abdominis plane blocks with epidural analgesia following upper abdominal surgery. Anaesthesia 2011; 66: 465-71.

13. Griffn J, Nicholls B. Ultrasound in regional anaesthesia. Anaesthesia 2010; 65(Suppl 1): 1-12.

14. Yoshida M, Furukawa T, Morikawa Y, Kitagawa Y, Kitajima M. The developments and achievements of endoscopic surgery, robotic surgery and function-preserving surgery. Jpn J Clin Oncol 2010; 40: 863-9.
15. Stiff G, Rhodes M, Kelly A, Telford K, Armstrong CP, Rees BI. Longterm pain: less common after laparascopic than open cholecystectomy. Br J Surg 1994; 81: 1368-70.

16. Wilson RG, Macintyre IM. Symptomatic outcome after laparascopic cholecystectomy. Br J Surg 1993; 80: 439-41.

17. Muktar K, Singh S. Transversus abdominis plane block for laparascopic surgery. Br J Anaesth 2009; 102: 143-4.

18. O'Connor K, Renfrew C. Subcostal transversus abdominis plane block. Anaesthesia 2010; 65: 91-2.

19. Chen CK, Phui VE. The efficacy of ultrasound-guided oblique subcostal transversus abdominis plane block in patients undergoing open cholecystectomy. South Afr J Anaesth Analg 2011; 17: 308-10. 